\title{
El Nuevo Papel de la Educación en el Funcionamiento de las Políticas Sociales en Cuba
}

Victoria Pérez Izquierdo(*)

Resumen: El presente artículo aborda el nuevo papel que se le concede a la Educación como esfera priorizada en el funcionamiento de la política social cubana y de ésta en el modelo de desarrollo trazado. Cuba apuesta al conocimiento para conseguir un crecimiento económico basado en servicios de mayor valor agregado, a diferencia de otros momentos del desarrollo cubano. Se presentan los resultados educacionales, no exentos de dificultades y limitaciones de recursos, y se observa la retroalimentación que hoy se produce a la economía a través de la exportación de servicios de salud, la producción de software y equipos médicos y la producción de productos biotecnológicos. Estas nuevas producciones y servicios, fruto del conocimiento de los profesionales cubanos, permiten acreditar cuantiosos ingresos a la balanza de pago cubana, sin dejar de prestar la colaboración solidaria y humanitaria a países de diferentes latitudes.

Palabras-claves: Educación, Gestión de la Educación.

\begin{abstract}
The present article enters upon the new role granted to the Education as a priority sphere in the Cuban social politics operation and in the pattern of development layout. Cuba bets to the knowledge in order to get an economic growth based on services of more added value, unlike to other moments of Cuban development. Educational results are submitted, not exempted of difficulties and limitations of resources, and the feedback which is taking place in the economy through the export of services of health, the software and medical equipament production as well as the biotechnical production. These new productions and services, fruit of the knowledge of Cuban professionals, allow to credit considerable revenues to the Cuban balance of payments, without stopping to lend the solidary and humanitarian collaboration to countries of different latitudes.
\end{abstract}

Keywords: Education, Management of Education.

$(*)$ Economista, investigadora del Instituto Nacional de Investigaciones Económicas de Cuba — INIE. E-mail: romelio@ tm.minbas.cu. Recebido em 4.9.09 e aceito em 29.10.09. 


\section{INTRODUCCIÓN}

Mostrar el impacto de la política social en Cuba, su implementación y adecuaciones en el nuevo contexto de desarrollo económico es sin dudas un orgullo para los cubanos. La prioridad otorgada a la política social dentro del modelo de desarrollo seguido, gracias a la voluntad del gobierno de mantener los logros sociales alcanzados, ha sido la clave para avanzar hacia los niveles de bienestar superiores que hoy se aprecian en la sociedad.

Al mismo tiempo, el desempeño realizado en las esferas sociales constituye objeto de interés no so solo para latinoamericanos sino para especialistas y ciudadanos de países europeos y asiáticos. Todos se preguntan como un país pequeño, bloqueado y de escaso desarrollo económico, ha podido alcanzar índices sociales similares al de países industrializados del primer mundo. La respuesta es simple, con voluntad política de gobierno es posible comprender los fuertes nexos que se dan entre las políticas económicas y las sociales y la influencia de lo social para incrementar el desarrollo económico, y que al mismo tiempo los ciudadanos reciban con justicia y equidad, mayor bienestar. Cuba ha apostado a su capital humano, formado por más de 40 años y en el nuevo milenio ya esta es la esfera que retroalimenta la economía a partir de su influencia en el resto de las ramas económicas y sociales. Es el conocimiento el motor impulsor de la economía de la sociedad cubana contemporánea.

La ponencia aborda de manera muy sintética una panorámica de la política social en los últimos años, su implementación y principales resultados. Igualmente aborda la esfera educacional, sus resultados y desafíos y el nuevo papel que se le concede en el modelo de desarrollo, como la nueva locomotora que impulsa la economía, tanto en el plano interno como para el ámbito internacional.

El conocimiento en el mundo moderno constituye el arma estratégica para enfrentar el desarrollo y Cuba lo intenta con optimismo a pesar de ser un país pobre, pequeño y bloqueado.

Por último, se señala que a pesar de los amplios resultados que se pueden mostrar en la esfera social, aun hay desafíos y retos que afrontar, ya que unas esferas han avanzado más que otras y en la medida que los problemas que hoy se puntualizan puedan ser resueltos, la población estará en mejores condiciones para afrontar el reto económico, que exige de personas sanas, nutridas, calificadas, y con un mínimo de condiciones habitacionales y de confort resueltas.

\section{Concepcion de la POLÍtica SOCIAL CUbANA}

La política social cubana desde el triunfo revolucionario de 1959, implicó un cambio sustancial para la población cubana que tenía grandes carencias sin esperanzas de solución. La política trazada de acceso universal a los servicios sociales, con total gratuidad permitió en pocos años elevar considerablemente el bienestar de las familias en la sociedad. 
El modelo de política social de Cuba, única en el continente americano, sostiene que toda persona tiene el derecho a la satisfacción de sus necesidades básicas, no como consumidor sino como derecho ciudadano. Las personas no son vistas como usuarios o clientes y desde la propia Constitución se plantean cuestiones como el derecho al trabajo, a la atención de salud por medio de servicios médicos gratuitos, el derecho a la educación - también gratuita — en todos los niveles de enseñanza, y a ser protegido contra el desamparo ${ }^{(1)}$.

La política social cubana favorece los valores de la solidaridad, el compañerismo, la ayuda desinteresada en detrimento del egoísmo y el individualismo acentuados en las sociedades neoliberales modernas.

En Cuba, dicha política traza los objetivos y las vías para alcanzar mejores resultados en cada una de las esferas sociales: la educación, la salud, la alimentación, la vivienda, el transporte, el empleo, así como la seguridad y la asistencia social, entre otras. Al evaluar los resultados obtenidos por Cuba, debe tenerse presente el importante efecto de complementariedad entre estas esferas, así como la interrelación de ellas con la política económica en cada momento histórico, en particular en períodos de restricción de recursos materiales y financieros condicionados por la crisis interna y los efectos del contexto internacional.

En particular, se aplicó la estrategia de utilizar a la educación como pivote para el avance en el resto de las esferas sociales, pues los conocimientos adquiridos posibilitan la incorporación de la población como agentes activos de los restantes proyectos sociales (FERRIOL, THERBON, CASTIÑEIRAS, 2005). En los últimos años en adición a las áreas mencionadas se destaca la seguridad y asistencia social, al contar Cuba con una población envejecida.

También debe resaltarse el énfasis otorgado a la equidad en la política social cubana, entendida no sólo como que la totalidad de la población tenga iguales oportunidades de acceso a la satisfacción de necesidades, sino también en el sentido de brindar mayores oportunidades de acceso a grupos sociales en posición desventajosa que les imposibilite aprovechar las oportunidades existentes.

Medidas redistributivas permitieron dar tratamiento preferencial a la mujer, los niños, ancianos y la población de zonas rurales, con el propósito de que la desigualdad resultante se mantuviera dentro de límites considerados admisibles para la sociedad.

En ocasiones las decisiones han privilegiado objetivos sociales por encima de otros exclusivamente económicos, y esta decisión se asume como un elemental acto de justicia ciudadana.

En especial la planificación ha posibilitado la compatibilización del complejo entramado de acciones que componen la política social, tomar en consideración lo territorial y dirigir los recursos financieros hacia los objetivos priorizados en la política social en cada etapa. La estabilidad del gobierno, unido a una sociedad en que la violencia no es una constante y se transpira seguridad ciudadana, han posibilitado la continuidad de dicha política, su seguimiento, evaluación y adecuación a cada etapa del desarrollo y más

(1) Constitución de la República de Cuba. La Habana. 1997. 
recientemente la implementación de numerosos programas que perfeccionan los servicios sociales que la población recibe y de medidas específicas que implican el aumento del bienestar familiar.

Respecto al financiamiento, el Estado cubano es el máximo responsable de garantizar la estabilidad en la prestación de los servicios sociales y ajustar la política social en cada momento en función de los intereses de la población, la cual participa activamente en el proceso de cambios y toma de decisiones. La Asamblea Nacional del Poder Popular (Parlamento) es el órgano máximo de poder; y es el único órgano constituyente y legislativo en la República. Desde 1995 la ejecución del presupuesto se ha descentralizado hacia los territorios.

Un rasgo distintivo de la política social es que se involucra en su cumplimiento no sólo a los ministerios rectores y otras instancias de la administración pública sino que participan las organizaciones políticas, sociales y las familias, lo que crea un entramado de actores que potencia la efectividad de las acciones, en torno a objetivos comunes, aportando cada cual sus fortalezas a través de su participación en el diseño de las políticas, en su aplicación y en su evaluación.

Los ciudadanos disponen de canales para hacer llegar sus insatisfacciones con los servicios. A esos fines pueden emplear los canales políticos, que comienzan con sus planteamientos al Delegado del Poder Popular de su localidad, y su curso hacia las instancias municipal, provincial y nacional de ser necesario. Pueden también utilizar vías directas a través de quejas ante proveedores y directivos de las instalaciones que prestan los servicios (INIE, PNUD, CEPAL, 2004).

Al culminar un quinquenio del nuevo milenio se aprecia que las modificaciones en la política social han estado condicionadas por los necesarios cambios en el modelo económico, al mismo tiempo que su accionar ha permitido alcanzar los niveles de crecimiento económicos que muestra Cuba. No sólo se han mantenido los principios de gratuidad y universalidad, sino que paralelamente se ha desarrollado un enfoque más activo en la identificación de las personas más necesitadas, lo que ha requerido flexibilidad y búsqueda de formas de gestión más dinámicas (INIE, 2005).

En los últimos años la atención personalizada, la elevación de la calidad de los servicios que recibe la población y el incremento del acceso, la equidad y la justicia social son las principales transformaciones de la política social cubana. Para ello, la nueva concepción dada en llamar "La Batalla de ideas" desencadena más de 200 programas sociales en las diferentes esferas sociales que de forma integrada apuntan hacia los objetivos señalados.

No obstante lo alcanzado quedan retos de consideración en esferas importantes del bienestar familiar como son la alimentación, la vivienda, los servicios personales, el transporte de pasajeros, entre otras. Lograr la integralidad y complementariedad de todas las esferas no es tarea fácil, pero lo logrado hasta el presente es una muestra de lo que puede hacer un país de escasos recursos financieros y tecnológicos, pero de un considerable capital humano, donde el gobierno se propone como objetivo de su política social alcanzar cada vez una sociedad más justa y dar respuesta creciente a las demandas de sus ciudadanos. 


\section{Principales Resultados de la POlítica social}

Con las medidas aplicadas desde el triunfo revolucionario se logró transformar la situación social heredada y avanzar sustancialmente en el bienestar popular, con justicia social. A partir de 1989, el modelo social estuvo sometido a los mayores retos desde su implantación. En primer lugar el déficit de recursos impactó severamente, en un inicio por limitaciones en los insumos para la prestación de los servicios y posteriormente el síntoma más claro de las dificultades fue el gradual deterioro de la infraestructura.

En segundo lugar, algunas de las medidas macroeconómicas de la reforma y otras de política económica, como la aplicación de una dualidad monetaria y el mayor espacio otorgado al mercado en la distribución tuvieron repercusiones directas en el funcionamiento de la sociedad, incluyendo la ruptura del canal de movilidad social anterior. La crisis económica agudizó algunas dificultades del modelo social antes descrito que fueron detectadas en la segunda mitad de los años ochenta, tales como limitaciones del sistema salarial como estímulo laboral y cierto grado de igualitarismo en el acceso al consumo.

Las primeras medidas de política social adoptadas en 1990, fueron la referida a mantener a los trabajadores en sus empleos y conservarles sus ingresos nominales aún en la etapa de contracción abrupta de la economía e ir realizando un ajuste gradual del empleo enmarcado en un proceso de redimensionamiento empresarial; garantizar los ingresos nominales a todos los pensionados por el sistema de seguridad y asistencia social; la decisión de trasladar la casi totalidad de los bienes de consumo disponibles al sistema de racionamiento para tratar de que la distribución fuera lo más equitativa posible; y la voluntad de preservar al máximo los programas sociales de salud y educación.

Posteriormente, las medidas sociales mostraron una tendencia, más profunda, de adaptación hacia la búsqueda de una política social más eficiente (INIE, 1998). Pueden distinguirse dos etapas. Una a partir de 1993 y hasta el año 2000, en que dicha búsqueda de eficiencia, expresada explícitamente en el propósito de mantener los logros sociales a pesar de la contracción de los recursos para el desarrollo social, abarcó algunas variaciones y posposiciones en los objetivos de la política social, transformaciones en el diseño de los sistemas de prestación de servicios, descentralización, modernización y fortalecimiento de las instancias locales y nuevos matices respecto a la participación de instituciones sin fines de lucro en la gestión y financiamiento de la política social.

En ese período, los objetivos de la política social en general se trataron de conservar, no obstante el elemento más revelador de que se transitaba por una nueva etapa, fue quizás observable en la política de empleo. Ésta, concebida como el pleno empleo y con financiamiento sólo estatal, fue variando hacia otra más flexible en que se admitía la existencia de un cierto nivel de desempleo y se reforzaba el papel del Estado en la protección del desocupado, todo lo cual tuvo connotación en la magnitud de los ingresos de la población.

En materia de descentralización y diversificación de los agentes que hacían la política social, se aplicaron mecanismos de gestión en que la escuela y el hospital adquirieron un papel más protagónico en el control de recursos, se iniciaron y desarrollaron 
programas de autoabastecimiento alimentario municipal, se descentralizó hacia las comunidades las acciones en vivienda, entre otras transformaciones. Las organizaciones sociales en la comunidad y las familias incrementan su iniciativa y participación en la revitalización y recuperación de los proyectos sociales.

También comenzaron a aplicarse nuevos instrumentos de financiamiento como fueron la prestación de servicios médicos a ciudadanos extranjeros, la docencia internacional de postgrado, entre otros. En sentido general se promovió el autofinanciamiento con recursos propios. Esta etapa de adecuación de la política social puede caracterizarse en general por la búsqueda de eficiencia y de nuevas fuentes de financiamiento.

Entre el año 2000 y el 2005 la política social enfatiza, por un lado, en la incorporación de nuevos objetivos estratégicos de desarrollo social - a pesar de las dificultades económicas aún existentes - al entenderse que sin trabajar en esas líneas la sociedad quedaría irremisiblemente apartada de la posibilidad de desarrollarse acorde a las características del mundo globalizado.

Surgen así objetivos de largo alcance como los de informatización de la sociedad y elevación de la cultura general de la población. Lo significativo es que, a pesar de las limitaciones de recursos de la economía, los nuevos programas se proponen brindarles oportunidades a todos sobre la base de aminorar los costos.

Por otro lado, a partir del año 2000 la política social adopta un carácter más personalizado. Sin renunciar a su universalidad, se inician una serie de acciones para evaluar mejor la situación del beneficiario potencial, seleccionarlo por esa vía y conocer su situación particular o su estado de necesidad, con vistas a atenderlo más adecuada y eficientemente.

Un eje central de la nueva concepción lo constituye la institucionalización del trabajador social, cuya función es detectar y evaluar los problemas de las familias en su comunidad de trabajo. En otro orden de cosas, hay aspectos del modelo y de la política económica más reciente que han impactado la sociedad cubana en diversas esferas, pero en especial en cuanto a la equidad. Los más importantes son la dualidad monetaria y la segmentación de mercados de bienes y servicios de consumo ${ }^{(2)}$.

En los últimos años, los programas sociales implementados en cada una de las esferas del desarrollo social han condicionado el mejoramiento de las condiciones de vida de la población a partir de un incremento en la calidad de los servicios prestados, una atención más efectiva y personalizada y un aumento de la equidad social, ante las no deseadas desigualdades de ingresos existentes en la sociedad.

A grandes rasgos, estas serían las principales modificaciones que ha sufrido la política social cubana, a lo largo de estos años, la cual se ha tenido que adaptar a las nuevas condiciones de un mundo más globalizado, ser más eficiente y personalizada, donde no se ha renunciado a los resultados obtenidos y aún con limitaciones econó-

(2) Se ha denominado mercado segmentado a aquel, en que se forman estratos donde rigen distintas formas de acceso para los compradores y vendedores, distintas reglas de formación de los precios, se utilizan diferentes monedas para las transacciones y, en general, difieren los mecanismos para captar los márgenes que se generan. 
micas, se propone objetivos ambiciosos, ya que el bienestar del hombre es su objetivo primordial. A continuación lo sucedido en las diferentes esferas de la política social cubana.

\section{LA ESFERA EDUCACIONAL}

Dentro de la amplia gama de transformaciones sociales que a partir de 1959 se inician en Cuba, la educación juega un papel importante en la estrategia del gobierno para lograr una sólida formación técnica-profesional y ética de la población. Desde los años 60 , se trazan acciones que reducen el analfabetismo ${ }^{(3)}$ y se crea el Sistema Nacional de Educación, con acceso universal y gratuito para todos los ciudadanos, sin discriminación por sexo, raza o estrato social.

Se desarrollaron acciones para elevar masivamente la escolaridad. La campaña para alcanzar el sexto grado en los 70'y durante los años 80, con la consolidación del Sistema Nacional de Educación, se trabajó por lograr los nueve años de estudio en toda la población. Un objetivo de la política educacional ha sido alcanzar niveles de desarrollo equitativos en los diferentes territorios, aspecto que se perfecciona en la actualidad con la aplicación de políticas y programas educativos de amplia participación de la sociedad civil para alcanzar metas superiores.

Durante la década de los 80', se adoptaron nuevas directrices, entre ellas, el incremento en la formación de recursos humanos e infraestructura necesaria para mantener la cobertura universal de la educación, y mejorar la calidad en la formación de los profesionales necesarios para el desarrollo económico; con la adaptación de los curriculum.

A principios de la década de los 90' el sector, al igual que el resto de la economía, sufre los efectos de la falta de recursos materiales y financieros sobre todo divisas para hacer frente a retos, que pueden catalogarse de ambiciosos para un país en crisis económica. Se mantienen los principios de la enseñanza (el carácter masivo de la educación, su gratuidad, la combinación del estudio con el trabajo con fines educativos, la coeducación que permite el acceso a la enseñanza por igual a niñas y niños y el principio de la participación de toda la sociedad en las tareas de la educación) y no-solo se mantiene lo logrado sino se avanza en términos de mejorar la calidad de la formación.

Adicionalmente, el sector ha desarrollado una política de perfeccionamiento organizativo y de funcionamiento en el logro de una mayor equidad en la prestación de los servicios, que posibilita alcanzar una mayor eficiencia económica, atendiendo a la diversidad y permite potenciar al máximo los recursos materiales, financieros y humanos existentes.

Una de las medidas más importantes en la descentralización fue dar mayor autonomía a la institución escolar y a su dirección, para el desempeño de sus funciones. A partir de la aplicación de esta política se observan mejores resultados ya que se conforman una unidad de intereses en cada centro escolar.

(3) Durante el año 1961 se realiza la campaña nacional de Alfabetización con carácter masivo, con amplia participación popular y se dicta la Ley de Nacionalización de la Enseñanza que apunta la responsabilidad del Estado en la prestación gratuita de los servicios educativos. 
De igual forma, ante los nuevos cambios ocurridos en la economía y en el entorno internacional, se han modificado los programas de estudio, con la incorporación de nuevas asignaturas y la ampliación de los perfiles de especialización, con la concepción de formar especialistas integrales que puedan ser ubicados en diversas plazas.

La política de recalificación y superación del personal docente, tanto de nivel superior como medio ha sido una preocupación constante del sistema. Al respecto, se desarrolla un fuerte despliegue de cursos de postgrados, maestrías y entrenamientos de los docentes en ejercicio; así como se ha jerarquizado que los maestros primarios cursen estudios universitarios de licenciatura en su especialidad, donde se alcanza el $70 \%$ con este título.

Estas fueron, entre otras, las principales políticas del Sector y las estrategias de adaptación desarrolladas por la educación cubana, a mediados de los 90', para enfrentar las restricciones de recursos, la reducción de las importaciones y el recrudecimiento del bloqueo económico sobre la Isla. La educación también se inserta en el proceso de reforma económica que tuvo lugar en el país.

Ya a partir de 1997, las transformaciones en la educación se orientaron a solucionar problemas de calidad, sin renunciar a su universalidad; a mejorar la relación entre la unidad del sistema educacional y la diversidad en que éste se manifiesta; vincular adecuadamente la centralización y la descentralización administrativas; crear en el magisterio un modo científico (objetivo, creativo y participativo, basado en investigaciones) de solucionar los problemas educacionales presentes y perspectivos y a la optimización del proceso docente.

A partir del año 2000, se señala que el objetivo principal de la educación es elevar el desarrollo humano de todos los cubanos, al propiciar una cultura general e integral desde la infancia; y promover la asimilación de conocimientos sólidos y profundos. Se enfatiza que se educa para la vida, a partir de valores acordes con el modelo social cubano, prestando atención a la diversidad de los alumnos, e integrando en ello a los propios estudiantes, a las instituciones y el personal de las escuelas, a la familia, a las instituciones y el personal de salud, a las organizaciones sociales y a los trabajadores sociales.

Para darle cumplimiento a estos aspectos, se están implementando programas educativos y sociales de corto y largo alcance que buscan desarrollar las aspiraciones y aumentar las oportunidades de cada niño o niña, adolescentes, y adultos para el acceso a la educación, el empleo, la salud, la cultura, etc. Los objetivos de estos programas son estratégicos, por su impacto en el nivel general de conocimientos, ampliación de la cultura y calificación de la población; e inciden positivamente en las condiciones de vida (ÁLVAREZ, 2003).

Se trabaja por mejorar la calidad de la enseñanza Primaria, con grupos de clases de no más de 20 alumnos y la reparación y ampliación del número de aulas con ese propósito. En consecuencia se comenzó la formación emergente de maestros primarios para asegurar el personal docente en estas escuelas, especialmente para los territorios que no lograban satisfacer tales necesidades. De igual forma, se dotaron de computadoras, televisores y videos a todos los centros docentes; se electrificaron por diferentes alter- 
nativas todas las escuelas primarias que aún no disponían de esta posibilidad; se trabaja en la producción de softwares y videos educativos para todos los niveles de enseñanza y en la creación de centros especializados para ello; y se editaron obras de consulta y de literatura para las bibliotecas escolares y en particular para los docentes.

El avance cualitativo de la enseñanza Secundaria Básica es también un objetivo y ha recibido los efectos de una serie de programas educativos. La reparación de escuelas ha sido básica, por el deterioro existente en la red docente. Durante los años 2001 y 2002 se ampliaron las capacidades en las escuelas, con el objetivo de brindar la doble sesión en todas las escuelas de esta enseñanza, meta que se materializa en el curso escolar 2003-2004.

La formación de un profesor integral para la educación Secundaria Básica (del séptimo al noveno grado de la enseñanza general); es otra transformación sustancial, pues permitirá el descenso del número de alumnos por aula a 15 y una atención más directa del maestro al alumno. La dotación de televisores y vídeos ha hecho posible la utilización del canal educativo, como apoyo al trabajo del profesor. El mejoramiento de los currículos docentes y el incremento de la computación son también aspectos que contribuyen a mejorar la calidad de esta enseñanza, así como la capacitación del personal docente.

Para los jóvenes que por diversos motivos no continuaron estudios medios se inician programas que permiten su reincorporación a las aulas. Comenzaron los cursos de superación integral para jóvenes de 17 a 29 años de edad, como una forma especial de empleo, así como los cursos para trabajadores sociales, profesores emergentes de primaria y profesores integrales de secundaria básica. Ellos disponen de los recursos adecuados tanto de medios audiovisuales como de bibliografía actualizada. Al concluir su preparación curricular de nivel medio podrán optar por carreras universitarias o por empleos acordes a su nueva calificación.

En la educación universitaria se aprecia un número de programas novedosos que sin dudas repercutirán en fomentar un capital humano cualitativamente superior. En primer lugar con el programa de la Universalización de la educación superior se pretende descentralizar aún más la formación universitaria en un grupo de especialidades, hacia los diferentes territorios del país e incrementar las oportunidades de acceso. Esto implicó la creación de sedes universitarias en todos los municipios del país con la utilización de instalaciones del nivel medio de enseñanza en horarios alternativos, y con profesores universitarios y otros profesionales a tiempo parciales residentes en la comunidad, debidamente categorizados para esta actividad docente, y dispuestos a apoyar el programa.

A través de la universidad del adulto mayor para personas de la tercera edad, jubilados y amas de casa que tienen interés de continuar estudiando, se vinculan al estudio y se sienten útiles a la sociedad a partir de diferentes actividades que realizan.

La creación de las bases que permitan la informatización de toda la sociedad es también uno de los programas más novedosos. Se han incrementado los medios técnicos destinados a los niños y jóvenes desde las edades más tempranas y en todas las enseñanzas con la consiguiente preparación acelerada de unos 20 mil docentes para dichos servi- 
cios y la habilitación en el uso de estas tecnologías a todos los maestros y profesores del país; se ha multiplicado en siete veces en los últimos cuatro cursos la formación en estas especialidades de la educación tecnológica; así como en la educación superior se incrementó la cantidad de centros universitarios que imparten la especialidad de informática. En adición, comenzó a funcionar la universidad de Ciencias Informáticas, con la dotación tecnológica requerida, con el objetivo de acelerar la formación de profesionales de nivel superior de esta especialidad. Se amplió y completó la red nacional de Joven Club de Computación ${ }^{(4)}$ en todas las comunidades, para el desarrollo de estas habilidades.

De igual forma y como ayuda técnica y financiera de otros países, Cuba ha fundado dos centros universitarios internacionales para la formación de médicos y de profesionales del deporte, otorgando becas a estudiantes, fundamentalmente latinoamericanos y africanos.

Por último, el objetivo más ambicioso es lograr el incremento de la cultura general integral de toda la sociedad. Para ello se creó un Canal Educativo con programación para los escolares, los jóvenes y otros dirigidos a toda la población adulta, y se promueve la cultura comunitaria a través de salas de vídeo, casas de cultura y centros comunitarios de computación. El programa televisivo Universidad para Todos, transmite cursos sobre la ciencia y la tecnología, el medio ambiente, el arte y la literatura, la historia universal, idiomas, y geografía, entre otros. Esto permite ampliar y actualizar los conocimientos de los diferentes segmentos poblacionales.

Como se aprecia, las estrategias y políticas desplegadas para alcanzar los objetivos educativos han ido variando a lo largo de estos años, con un énfasis en aprovechar el potencial existente en el país, y preparar a la población para contextos globalizados, donde la ciencia y la técnica tendrán un espacio esencial, y el desarrollo económico tendrá su base en el conocimiento adquirido.

\section{Resultados de la POLÍtica eduCACional}

A pesar de las difíciles condiciones económicas afrontadas por el país en los años 90', el sector educacional ha sido priorizado por el Estado cubano, de manera que no sólo se mantiene la gratuidad en la prestación del servicio educativo y el acceso a todos por igual, a partir de una alta cobertura en la enseñanza básica, sino se han alcanzado índices superiores (PÉREZ, 2000). Los resultados que hoy se aprecian, son muestra de la voluntad explícita para hacer de la educación un pilar fundamental para el desarrollo económico y social.

La escolaridad en Cuba alcanza como promedio 9 años de estudios, y se observa un bajo por ciento de analfabetismo residual. El índice de escolarización apunta que el $99,5 \%$ de la franja de 6 a 11 años de edad recibe los servicios educacionales. Este indicador para el grupo de 6 a 14 años que incluye la enseñanza media básica es del 98,6\%, mientras que la escolarización del grupo de 6 a 16 años asciende al 95,6\%.

(4) Fueron construidos en todos los municipios del país esta red de centros para que desde edades tempranas, en la comunidad, los niños y jóvenes tengan acceso a la computación. 
La equidad en la educación se plantea no sólo en dar igualdad de oportunidades a todos, sino en obtener cada vez resultados menos diferenciados. Muestra de ello son los elevados índices de escolaridad que se aprecian en los diferentes territorios cubanos.

En cuanto a la calidad de la enseñanza cubana, se observa que Cuba fue el país que obtuvo los resultados más altos en el ámbito regional en un estudio internacional realizado por el Laboratorio Latinoamericano de Evaluación de la Calidad de la Educación, coordinado por la UNESCO, durante 1997. El estudio, realizado en 12 países latinoamericanos evaluó los conocimientos de los alumnos en las disciplinas de Matemática y Lenguaje y analizó los factores asociados al rendimiento escolar. Los resultados alcanzados distan mucho de lo alcanzado por los países de la región, no solo se alcanzó el primer lugar en un conjunto de índices, sino la brecha entre el país más cerca fue sustancial. Con posterioridad los estudios realizados sobre el aprendizaje escolar muestran que la utilización de los medios audiovisuales, de la multimedia y la aplicación de métodos de enseñanza interactivos, han propiciado resultados sostenidos en la calidad de la educación (RODRÍGUEZ, 2005).

La continuidad de estudios, esta prevista en el sistema de educación, permitiendo no solo el paso de los alumnos de unos subniveles a otros, sino el rescate de los estudiantes que causan baja en alguna enseñanza y su incorporación a otras.

El tratamiento de la educación discapacitada en Cuba merece resaltarse. A través de la Educación especial, los niños y jóvenes que presentan deficiencias físicas, mentales y trastornos de conducta, se incorporan en lo posible a la sociedad y no se encuentren marginados socialmente. Cuba muestra avances en esta sensible labor. Esta enseñanza ha sido prioridad en las líneas estratégicas de trabajo del Sistema Nacional de Educación, y se ha mejorado la dotación técnica de los centros. Los programas en curso permitirán la detección de los niños aún no atendidos y brindarles este servicio de mayor calidad. La cantidad de niños matriculados asciende a más de 50 mil, existiendo 435 instalaciones de las 11 especialidades existentes ${ }^{(5)}$.

La educación tiene como reto el formar a los profesionales de todas las disciplinas, prepararlos para la vida y para las exigencias que el desarrollo económico demanda, al igual que forma a sus propios profesionales. El momento más crítico con los profesores fue en el curso escolar 1993-94, año en que el éxodo fue el $10 \%$ de la fuerza docente, a partir del deterioro de las condiciones de trabajo, el aumento de los problemas cotidianos, y la búsqueda de incentivos en otras actividades de la economía. Posteriormente, con la recuperación paulatina de la economía, el incremento salarial y la estimulación material y moral a los profesionales, comenzó nuevamente a elevarse él prestigió del profesor, lo que condicionó una disminución del éxodo de profesores y maestros y un retorno de muchos al sector de educación.

El personal docente creció significativamente durante el periodo 1997-05, a partir de la reducción de alumnos por aula, y otros programas en ejecución. En el curso acadé-

(5) Existen 261 escuelas de retraso mental, 85 del retardo físico, 41 de trastornos de la conducta, 16 de sordos, 20 de ciegos y estrábicos, 9 de trastorno en el lenguaje, de impedidos físicos, de autismo y rehabilitación del Asmático y el Diabético; todas han tenido un fuerte apoyo de recursos para la compra del equipamiento especializado necesario para mejorar la calidad del servicio. 
mico 2004-05, se ejercieron un total de 263,3 miles de profesores en centros docentes, de los cuales frente al aula están más del $87 \%$. El 56\% de los profesores se encuentran ubicados en la enseñanza Primaria, el 33\% imparte docencia en la enseñanza media y la educación superior agrupa al $11 \%$ de los docentes del sistema. A la cifra de profesores universitarios hay que añadir una cantidad importante de profesionales que se contratan para impartir clases en la Universalización de la Educación Superior en los municipios del país.

Se debe apuntar que el 100\% del cuerpo docente que se desempeña en el nivel primario están titulados y más del $70 \%$ de ellos son licenciados en educación; en el resto de los niveles es más del 95\%, lo cual fue posible gracias a la red de instituciones superiores pedagógicas para la titulación, recalificación y superación de los maestros. Durante el 2005 se aumentaron los salarios de los trabajadores de la educación, estimulándose particularmente la obtención de grados científicos.

En cuanto al financiamiento, el Estado cubano es el máximo responsable, ocupando un lugar prominente el gasto corriente de educación dentro del presupuesto. Los gastos corrientes de educación en el total de gastos corrientes de la actividad presupuestada del país ascienden a un $18 \%$ y con relación a los gastos totales del presupuesto alcanzan un $17 \%$. Como porcentaje del PIB, los gastos corrientes de educación alcanzaron en el 2004, un 9\% (ONE, a, 2005).

A partir del año 1998 se aplica una reforma que incrementó los salarios de los trabajadores que laboran en el sistema de educación. El salario mensual que reciben los docentes está determinado por los siguientes elementos: el nivel de calificación profesional, el tipo de centro donde labore, los años de servicios prestados en la docencia, el cargo que desempeña y los resultados de la evaluación. El sistema de estimulación salarial, se basa en los resultados de la evaluación del maestro y los rendimientos obtenidos. Esto implicó un costo cercano a los 250 millones de pesos. Recientemente, se incrementan nuevamente los salarios de los educadores, lo que implica un prestigio mayor a la profesión y un estimulo económico al personal del sector. Dicho aumento ha beneficiado específicamente a 470,6 miles de trabajadores de la educación preescolar, media y superior y ha significado ampliar cada año los gastos de salario en 260 millones de $\operatorname{pesos}^{(6)}$. No obstante, estos incrementos salariales, para algunos profesionales aún son insuficientes para el elevado costo de la vida actual ${ }^{(7)}$.

\section{PrinCipales desafíos de la educación}

La educación en Cuba continúa siendo una de las esferas priorizadas dentro del desarrollo social, a pesar de las actuales limitaciones de recursos, que impone el desarrollo económico y la adversa situación internacional de estos tiempos.

(6) Consultar Economics Press Service, año 18, n. 22, 30 de noviembre de 2005.

(7) La propia situación económica explicada anteriormente, la doble moneda, la incursión en diferentes mercados y los elevados precios de productos y servicios para aquellos que no reciben dólares implica diferentes contextos antes los incrementos salariales experimentados. 
A pesar de los avances, persisten problemas, sobre todo al considerar la educación como sector estratégico para impulsar el desarrollo económico del país y considerar la formación como un proceso continuo de perfeccionamiento.

Los programas implementados constituyen un desafío para el modelo cubano, pues su aplicación masiva implica una utilización eficiente y un incremento sustancial de la calidad en los diferentes niveles de la formación.

La retribución de los docentes y su mayor reconocimiento en la sociedad constituyen aspectos contemplados en la reforma de salarios en curso, su crecimiento estará en correspondencia con los niveles de crecimiento económico que el país sea capaz de alcanzar y las decisiones políticas que al respecto se implementen. De igual forma, la recalificación de los docentes y el mejoramiento de los curriculum y programas de estudios, son objetivos permanentes del sistema nacional de educación en todas sus instancias, territorios y niveles de instrucción.

Cuba proyecta hacia el futuro su labor educativa desde la perspectiva de una profunda revolución educacional que se propone cambios en los conceptos y modos de hacer la educación y la elevación de los niveles ya alcanzados de cultura de todos los ciudadanos. La meta principal de los esfuerzos sociales del gobierno y el estado cubano es la de elevar los niveles de cultura de la sociedad cubana de forma permanente, como premisa para elevar el desarrollo económico.

En esta interrelación entre educación y desarrollo, el país ha puesto el énfasis en el capital humano, en las ventajas adquiridas (el conocimiento), de manera que los ciudadanos estén mejor preparados para enfrentar la era de la informática, en un mundo contemporáneo, competitivo, dinámico y globalizado y la educación se convierta en el arma estratégica en el tránsito a la economía del conocimiento. Ya durante el estos últimos años y hasta el 2008 se demuestra no sólo la colaboración de este personal en países necesitados, sino lo que puede ingresar el país a partir de la exportación de los servicios profesionales, intensivos en conocimientos.

\section{Conclusiones}

Desde inicios del nuevo milenio la política social cubana se ha ido adecuando a las nuevas condiciones económicas y sociales, no sólo para conservar los resultados satisfactorios anteriores sino con el objetivo de aumentar la calidad de la atención a la población y crear condiciones que en perspectiva contribuyan al desarrollo del país.

En el ámbito económico, se han mantenido limitaciones de recursos y otras afectaciones provocadas por el desempeño de la economía internacional y por dificultades internas que limitan el crecimiento. En esas condiciones la dinámica acelerada de recursos públicos dedicados a la política social evidencia que permanece en el gobierno la concepción de que el desarrollo social es condición necesaria al desarrollo. También se observa que la búsqueda de eficiencia en la política social se ha convertido en eje central de las transformaciones.

Por otra parte, la emergencia de problemas de vulnerabilidad, pobreza y desigualdad han obligado a que la política social tenga en cuenta en mayor medida que antes las 
diferenciadas situaciones de los beneficiarios potenciales y a que los beneficios lleguen en mayor medida a los más necesitados. A ello se añade el impacto del acelerado proceso de envejecimiento de la población que introduce nuevos desafíos a las acciones sociales.

Ambos elementos, déficit de financiamiento y necesidad de focalizar los beneficios sin abandonar la universalidad de la atención, han determinado que la política social haya continuado desarrollándose de modo creativo y cada vez más integral. El análisis de los nuevos programas sociales en ejecución a partir del año 2000 apunta líneas muy promisorias sobre cómo proceder para que se potencien las acciones y los resultados excedan las posibilidades financieras. La evaluación futura que podrá efectuarse sobre sus impactos puede aportar lecciones útiles para otros países en desarrollo.

Una serie de características de la sociedad cubana coadyuvan a que sea posible la aplicación de una política social como la descrita. En primer lugar, se cuenta con la voluntad política del gobierno sin la cual poco se puede lograr. A ello se añade el compromiso colectivo con la realización de un proyecto social, el elevado desarrollo social alcanzado previo a la crisis económica de los noventa, y la solidaridad que caracteriza a la población, todo lo cual se convirtió en una fortaleza para contrarrestar las severas dificultades.

En segundo lugar, Cuba es una sociedad con un elevado grado de institucionalidad, organizada, con capacidad de controlar, evaluar los resultados con criterios científicamente fundamentados, y monitorear las situaciones locales; y al mismo tiempo cuenta con un sistema de estadísticas económicas y sociales suficientemente desarrollado.

En tercer lugar, en la aplicación de las medidas sociales participan de conjunto el gobierno, las organizaciones sociales, las organizaciones políticas y las familias.

Sin embargo, para disminuir los problemas de pobreza, desigualdad y vulnerabilidad se requiere, además de la vía social, lograr avances en las condiciones económicas pues el crecimiento también es condición necesaria para el desarrollo social. Además, aunque se incremente la eficiencia de la política, los recursos pueden ser insuficientes para continuar programas tan abarcadores e integrales.

A esos propósitos parece conveniente que se brinde continuidad a la reforma económica aplicada a partir de 1993, en especial en esferas con impactos sociales elevados. Los propósitos de eliminar la dualidad monetaria, disminuir la segmentación de los mercados de bienes y servicios de consumo y reestructurar el mercado laboral deben formar parte de esa agenda.

De igual forma es aconsejable la revisión y evaluación periódica de los más de 200 programas sociales implementados, de forma tal que se midan los impactos generados en las poblaciones objetivo y su retroalimentación en la sociedad. En adición, dos nuevas esferas marcan nuevas prioridades a partir del 2006, al disponer de programas y recursos para su recuperación, ellas son la vivienda y el transporte de pasajeros. Su desempeño, alcance y satisfacción de las demandas constituirán un reto de la política social para el próximo quinquenio.

Dada esa panorámica de la Política Social cubana y en particular las políticas educativas como nueva locomotora de la economía es que la autora considera esencial com- 
prender el papel de las mismas en el modelo de desarrollo previsible para la economía cubana, ya que se han desarrollado concepciones muy propias, no exportables a otras latitudes y a pesar de sus logros, contradicciones y desafíos han situado a Cuba en un buen peldaño social, en el contexto Latinoamericano.

\section{Bibliografia}

ÁLVAREZ, Elena. Cuba: la coyuntura económica en el año 2002 y perspectivas para el 2003. Informe, INIE, 2002.

Desafíos para el desarrollo económico y social en los albores del tercer milenio: el caso de Cuba. Ponencia al VIII Congreso de la AEALC, 2000.

CONSTITUCIÓN DE LA REPÚBLICA DE CUBA. La Habana: 1997.

CUMBRE MUNDIAL SOBRE ALIMENTACIÓN. 2002.

DECRETO-LEY 141. Resolución Conjunta n. 1 CETSS-CEF, Resoluciones Conjuntas No. 3 y 4 , 1995. MTSS-MFP y Resolución n. 10, 1995 del MTSS, 1993.

ECONOMICS PRESS SERVICE. Crecen salarios, pensiones y tarifas eléctricas, año 18, n. 22, 30 nov. 2005.

FERRIOL, Angela; CASTIÑEIRAS, Rita; THERBORN Goran. Política social: el mundo contemporáneo y las experiencias de Cuba y Suecia. Uruguay: 2005.

FERRIOL, Angela; Anne, L.; XUAN, Hoang. Promoción de exportaciones, pobreza, desigualdad y crecimiento. El caso de Cuba en los noventa. Investigación, n. 1, enero/mar. 2003.

FERRIOL, Angela; QUINTANA, Didio; PÉREZ, Victoria; ATIENZA, Aída. Política social en el ajuste y su adecuación a las nuevas condiciones. Revista Cuba, Investigación Económica, La Habana, INIE, n. 1, 1998.

FERRIOL, Angela; QUINTANA, Didio; PÉREZ, Victoria; ATIENZA, Aída. Servicios de educación, salud, agua y saneamiento en Cuba. Documento elaborado para el Banco Mundial, 2003.

INIE, PNUD, CEPAL. Reformas estructurales y políticas sociales: Cuba a inicios del nuevo milenio. México: 2004.

INSTITUTO NACIONAL DE INVESTIGACIONES ECONÓMICAS. INIE. Transformaciones de la gestión Social en Cuba. Proyecto de investigación, colectivo de autores, publicación electrónica en CD de la Jornada Científica del INIE, 2005. Disponible en: <http://www.inie.cu>.

MEP. Informe sobre la situación de la vivienda en Cuba. 2003.

MINISTERIO DEJUSTICIA. Ley de seguridad social, regulamento, disposiciones complementarias. La Habana: Ministerio de Justicia, Titulo III, Capitulo 1, 1987.

MINSAP. Anuario estadístico, 2004.

Temas de estadísticas de salud. Encuesta de indicadores múltiples por conglomerados (MICS). Standard de UNICEF, 2002.

MORALES, Alfredo. Intervención en la II Asamblea Mundial sobre Envejecimiento. Madrid: 2002.

OFICINA NACIONAL DE ESTADÍSTICAS. Estadísticas seleccionadas 2004. Cuba: 2005a. Anuario estadístico de Cuba 2004. Cuba: 2005b.

PÉREZ, Victoria. La gestión de la educación: latinoamérica y Cuba. Cuba, 2004. Tesis (Maestría) — Instituto Nacional de Investigaciones Económicas, INIE. Disponible en: <http://www.inie.cu>. 
Ajuste económico e impactos sociales. Los retos de la educación y la salud pública en Cuba. Ponencia presentada a LASA 2000, XXII Internacional Congress. Miami: 2000.

RESOLUCIÓN 51 del Comité Estatal del Trabajo y Seguridad Social, del 1 enero. de 1989.

RODRÍGUEZ, José Luis. Discurso pronunciado por el Ministro de Economía y Planificación de la República de Cuba ante el 6to. Período Ordinario de Sesiones de la Sexta Legislatura de la Asamblea Nacional del Poder Popular. Suplemento especial de Granma, enero. Ciudad de La Habana: 2006. 